\title{
Zum Problem der Stabilität der Klimabedingungen in Westsibirien während der Glaziale und Interglaziale
}

\author{
Von W. WeIschet, z. Zt. Valdivia/Chile
}

Mit 2 Abbildungen im Text

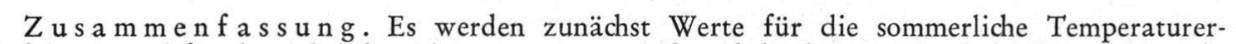
niedrigung und für den jährlichen Temperaturgang während der letzten Eiszeit in Westsibirien abgeleitet. Grundlage hierfür bildet die verschiedene Lage der Grenzen von Tundra und Laubmischwald zur Eiszeit (nach Frenzel \& Troll 1952) und in der Gegenwart (Sowjetatlas 1937). Das Ergebnis ist eine sommerliche Temperaturdepression von nur $5-8^{\circ} \mathrm{C}$ nahe dem Polarkreis und von $2-4^{\circ} \mathrm{C}$ in $50^{\circ}$ Breite gegenüber $10-12^{\circ}$ in Mitteleuropa.

Anschließend wird die Frage diskutiert, ob dies allein eine Folge der größeren Kontinentalität des zentralen Teiles Eurasiens gegenüber dem westlichen und östlichen Teil des Doppelkontinentes sein kann. Es stellt sich heraus, daß die relative Stabilität der Klimabedingungen Westsibiriens im ganzen Quartär sich zwanglos als Folge des Wechsels von vorherrschender Meridionalzirkulation im Glazial und verstärkter Zonalzirkulation im Interglazial und Postglazial erklären läßt.

$\mathrm{Sum} \mathrm{mary}$. The depression values of the summer temperature and also of the mean annual temperature in Western Siberia during the last glaciation, have been deduced. This calculation has been based on the different position of the boreal boundaries of the tundra and the continental deciduous forests during the ice ages and at the present time. According to these calculations the following depressions of summer temperature have been obtained: $5-8^{\circ} \mathrm{C}$ near the arctic circle and $2-4^{\circ} \mathrm{C}$ in the latitude of $50^{\circ}$ North, in comparison with the $10-12^{\circ} \mathrm{C}$ depression in Central Europe.

Afterwards it has been discussed whether or not these relatively small depressions can be explained exclusively on the basis of the higher continentality of the central part of Eurasia in comparison with the western or eastern part of this continent. As this seems to be impossible, it is necessary to look for other facts that could be related to this problem. In the opinion of the author the relative stability of climatic conditions in Western Siberia during the whole Quaternary could be easily explained by the change between the meridional-circulation (low-index-type), prevailing during the glacial periods, and the reinforced zonal-circulation (high-index-type) during the interglacial and postglacial periods.

Die Formulierung „Stabilität der klimatischen Bedingungen“ wurde in Anlehnung an Guerassimow \& Markow (1954) gewählt, die in einer zusammenfassenden Darstellung der bis dato erworbenen Kenntnisse über die Paläogeographie der Gebiete der Sow jetunion während des Quartärs auf dem Geologenkongreß in Algier in Bezug auf Westsibirien von "größter Stabiliät der geographischen Bedingungen" sprachen. Sie ist ein umfassendes Resumé gegenseitig sich stützender älterer Beobachtungen und Schlußfolgerungen und wird von später erschienenen Arbeiten noch erhärtet. Alle führen zu dem Ergebnis, daß die physiogeographischen Veränderungen, die mit dem Wechsel von Glazial und Interglazial die ganze Erde im Pleistozän betroffen haben, in West- und auch in Mittelsibirien von auffallend geringer Intensität und räumlicher Ausdehnung waren, besonders im Vergleich zu denjenigen der im Westen und Osten angrenzenden Teile des eurasiatischen Doppelkontinentes. Unter paläoklimatologischer Sicht bedeutet das: die pleistozänen Klimaschwankungen haben im zentralen Teil Nordeurasiens eine geringere Schwankungsweite gehabt als westlich und östlich davon. Zur Begründung dieses Tatbestandes liegt es bei Anwendung aktualistischer Gesichtspunkte zunächst nahe, ihn mit der unterschiedlichen Kontinentalität der genannten Räume in Verbindung zu bringen, wie es nach PARMUziN z. B. von Frenzel \& Troll (1952) sowie Parmuzin (1954) geschehen ist. Da die These aber den von Frenzel \& Troll behandelten Problemkreis nur am Rande berührt, wurden die Möglichkeiten der sich in den bezeichneten Gebieten auswirkenden größeren Kontinentalität und ihre Konsequenzen nicht weiter diskutiert. Doch erscheint es mir gerade im Zusammenhang mit den Fragen der Entwicklung und Herkunft der prähistorischen 
Menschheit von Nutzen zu sein, den Gedanken unter paläoklimatologischem Gesichtspunkt aufzugreifen und weiterzuführen. Der Beitrag möge meinem Lehrer C. TRolı anläßlich der Vollendung seines 60. Lebensjahres gewidmet sein.

\section{Die mögliche kaltzeitliche Temperaturdepression in den Sommermonaten}

Zunächst möchte ich versuchen, aus dem Vergleich der rezenten und kaltzeitlichen Verbreitung einiger wichtiger Pflanzenformationen in Nordeurasien eine Vorstellung von der möglichen sommerlichen Temperatur-Erniedrigung zur letzten Kaltzeit und vom damaligen jährlichen Temperaturgang im westlichen Sibirien abzuleiten.

Als Grundlage, in welcher die geomorphologischen, geologischen, glaziologischen und vor allem paläobotanischen und -faunistischen Klima-Indizien in einer zusammenfassenden Überschau ausgewertet sind, sollen die Karten von Büdel (1951) bzw. Frenzel \& Troll (1952) über die „Klimazonen zur Würmeiszeit“ bzw. die „Vegetationszonen des nördlichen Eurasiens während der letzten Eiszeit" dienen, punktweise ergänzt durch neuere Veröffentlichugnen von Funden oder Beobachtungen. Es muß unterstellt werden, daß sich an den Übersichtsdarstellungen mit der Ausweitung der Kenntnis in den Einzelheiten noch manches ändern kann; die Grundzüge sind aber unbestritten, und die in der vorliegenden Abhandlung zu wählenden speziellen Anknüpfungspunkte scheinen mir genügend durch Funde in ihrer räumlichen Lage und ihrer qualitativen Aussage gesichert zu sein, um Folgerungen anschließen zu können.

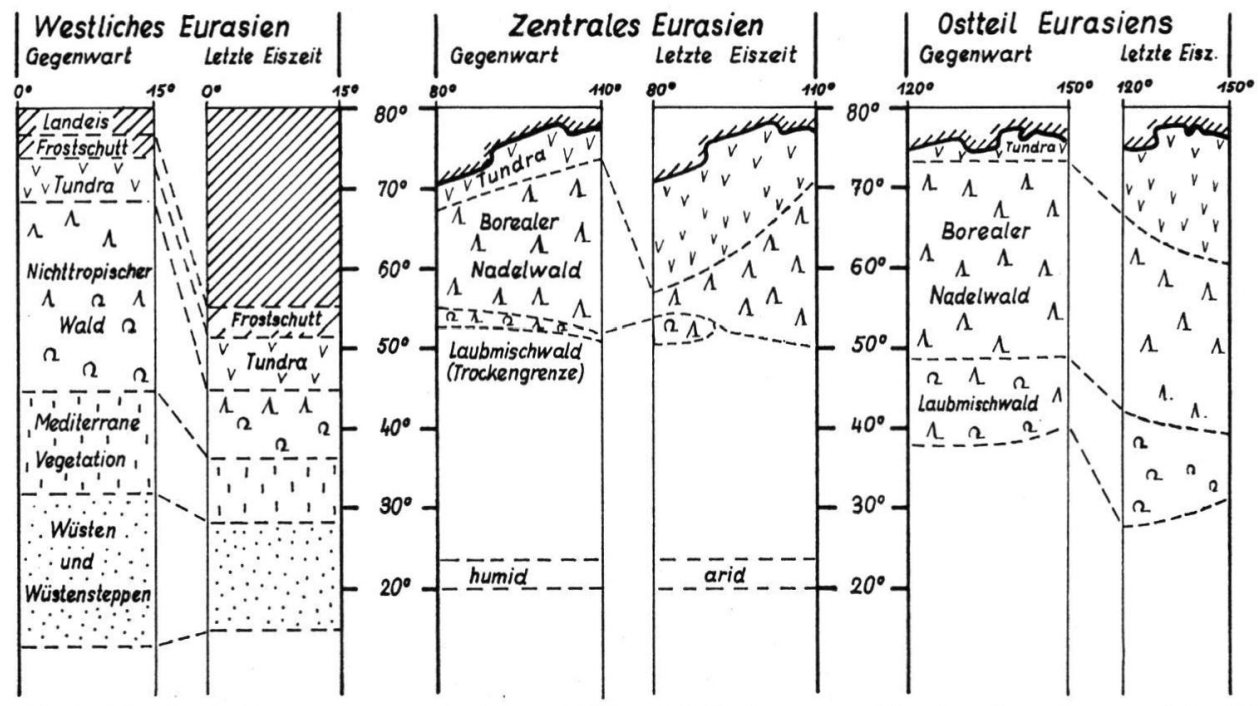

Abb. 1. Vergleich der rezenten und würmzeitlichen Verbreitung von Tundra, borealem Nadelwald und Laubmischwald im westlichen, zentralen und östlichen Eurasien (nach BüDEL 1951 und Frenzel \& Troll 1952).

In Abb. 1 sind für den westlichen ( $0^{\circ}$ bis $15^{\circ}$ Ost), mittleren $\left(80^{\circ}\right.$ bis $110^{\circ}$ Ost) und östlichen Teil $\left(120^{\circ}\right.$ bis $150^{\circ}$ Ost) Eurasiens die wichtigsten klimabedingten Vegetationszonen ihrer rezenten und würmzeitlichen Verbreitung entsprechend eingetragen, sowie der unterschiedliche Betrag der kaltzeitlichen Südverlagerung zur Darstellung gebracht. Dabei entspricht die Darstellung von Westeurasien den Angaben von BüDEL (1951, vgl. auch Woldstedr 1954), dessen Schema als Vorbild für die ganze Abbildung diente. In dynamisch-klimatologischer Ausdeutung besagt die Darstellung für Westeurasien folgendes: das unverrückte Widerlager der Anordnung der zirkulationsbedingten Klimazonen ist der Kern der subtropischen Hochdruckzelle etwas nördlich von $20^{\circ}$, repräsentiert durch 
den Wüsten- und Steppengürtel. Von Norden her wurde die labile Zone des ektropischen Westwindgürtels durch den Vorstoß des polaren Systems mit Landeis, Frostschutzzone und Tundra im Gefolge vorwiegend auf Kosten seines borealen Teiles (Nicht-tropischer Waldgürtel) zusammengedrückt und in abklingendem Maße nach Süden verschoben. Gleichzeitig gewinnt die innertropische Zirkulation polwärts gegen den Hochdruckgürtel an Raum, da dieser in seiner Wirkungsmöglichkeit geschwächt ist. Von Norden greift die intensivere Zirkulation der Subtropen, von Süden die ausgeweitete der Tropen auf ihn über. Er wird als Trockengürtel weniger extrem; landschaftskundlich werden die Wüsten von Steppengebieten durchsetzt (Ausdruck der Pluvialzeit in der subtropisch-randtropischen Trockenzone). Speziell wurde im Meridian-Ausschnitt West- und Mitteleuropas die Grenze zwischen baumfreier Tundra und nichttropischem Wald zur Eiszeit um 24 Breitengrade von ca. $69^{\circ} \mathrm{N}$ bis $45^{\circ} \mathrm{N}$, die Südgrenze des thermisch anspruchsvolleren Laubmischwaldes um 9 Breitengrade von $45^{\circ}$ bis $36^{\circ}$ im Mittel zurückgedrängt.

Im zentralen Teil des Kontinentes ( 80 bis $100^{\circ}$ Ost) hingegen verlief die Grenze derjenigen Vegetationsgebiete, in denen der Wald den Aufbau wenigstens mitbestimmte (Waldsteppe-Waldtundra, Wald- und Strauchtundra sowie borealer Nadelwald nach Frenzel \& Troll 1952) von ca. $56^{\circ}$ am Ischim nach ca. $70^{\circ}$ an der unteren Lena, also 15 bzw. $35^{\circ}$ weiter nördlich als in Mitteleuropa. Als Belege können folgende Befunde gelten: in der Nähe der paläolithischen Fundstellen im Umkreis von Krassnojarsk bei $56^{\circ}$ am Jenissei sind Birke, Lärche und Weide als Vertreter der Taiga-Hain-Vegetation nachgewiesen, und die Tatsache, daß im oberen Wiljuj-Gebiet zwischen den Zähnen eines Nashorns Holzfasern und Nadeln von Nadelbäumen gefunden wurden (THIEL 1951), zeigt wenigstens, daß in diesem Bereich Nadelbäume bis zum Polarkreis vorkamen. Eine Stütze erfahren diese Argumente in gewisser Weise durch die Feststellung, daß das nördlich des Polarkreises gelegene 1000 bis $2000 \mathrm{~m}$ hohe Norilsker Plateau oder auch das bis $2000 \mathrm{~m}$ hohe Byrranga-Gebirge der Taimyr-Halbinsel nur Talgletscher aufgewiesen haben, die Chatanga-Senke eisfrei war. Neuerdings glaubt zwar BLAGoweschtschensKaja (1955) vom Unterlauf der Unteren Tunguska (also ca. $63^{\circ}$ Nord) Spuren der Würm-Vereisung doch nachweisen zu können, die vorher Komanis und Kuschew bestritten haben. Aber es handelt sich wohl um eine mehr lokale Ausbildung, wie ja auch sonst nur vom Oberlauf des Wiljuj im zentralen Teil des Mittelsibirischen Berglandes lokale Moränen in $800 \mathrm{~m}$ Meereshöhe bekannt sind. Der ganze übrige Bereich des Mittelsibirischen Berglandes gilt mit Sicherheit für die Würmeiszeit als eisfrei, wie PARmuzin (1954) noch einmal zusammenfassend feststellt. Erst im Sajanischen Gebirge finden sich wieder Spuren kleiner Gletscher. THIEL (1951) gibt dort als Schneegrenzhöhe $2000 \mathrm{~m}$ an.

Im Vergleich zu rezenten Verhältnissen muß man für den zentralen Teil Eurasiens also mit einer Südwärtsverlagerung der baumfreien Tundra zur Würmeiszeit von nur 10 Breitengraden im westlichen, bzw. drei im ostwärtigen Teil rechnen.

Bezüglich der Zurückdrängung des thermisch anspruchsvolleren Laubmischwaldes sind die Beträge noch geringer. Die Südgrenze der borealen Nadelwälder lag ostwärts des Urals bei ungefähr $5^{\circ}$. Bei Dschilandschik (ca. $49^{\circ}$ ) in der Kirgisensteppe ist neben Fichte und Lärche auch die Eiche und damit eines der wärmebeanspruchenden Laubhölzer nachgewiesen. Frenzel bestätigte auf briefliche Rückfrage neuerlich, „daß sowohl florengeographische Überlegungen als auch pollenanalytische Untersuchungen die Existenz eines Waldes zur letzten Eiszeit zwischen oberem $\mathrm{Ob}$ und Ischim beweiskräftig darlegen". Nach dem erwähnten Fund des Eichenblattes und florengeographischen Zusammenhängen müsse man sich den Bestand als lockeren Eichen-Birken-Hain vorstellen. Die erwähnte Zone entspricht in der Breitenlage den eiszeitlichen Tundrengebieten in Mitteleuropa.

In Nordostasien ist dagegen wieder durch zahlreiche Funde die Ausdehnung der baumfreien Tundra nach Süden bis über den Polarkreis belegt. Durch Bohrungen, die von Neustadt \& TJulina (1936) ausgeführt wurden (zit. nach Frenzel \& Troll 1952) ist 
sie am Main, am Mittellauf des Anadyr in Breiten von 63 bis $64^{\circ}$ nachgewiesen. Die Vergletscherung dieses Bereiches war ebenfalls bedeutend stärker als diejenige Mittel- und Westsibiriens. Zwar war es auch keine Inlandeisdecke wie im atlantischen Westen, da selbst die Indigirka-, Kolyma- und Jana-Niederung eisfrei blieben. Aber die Beobachtungen deuten auf eine starke Gebirgsvergletscherung hin. Die Schneegrenze lag am oberen Kolyma ( 61 bis $62^{\circ}$ Nord) bei $600 \mathrm{~m}$, auf dem südlichen Kamtschatka bei $700 \mathrm{~m}$ (K $\mathrm{K}_{\text {LUTE }}$ 1928). Für den unteren Sela $\left(54^{\circ}\right)$ wird sie nach Nikolskaja \& Schtscherbakow (1956) mit $1500 \mathrm{~m}$ anzusetzen sein, nach von Wissmann (1938) in der Kalmi-Kette im Norden Koreas in $2200 \mathrm{~m}$ und im zentralen Hondo bei 2400 bis $2600 \mathrm{~m}$. Die zuletzt genannten Gebiete liegen aber schon etwas nördlich bzw. südlich des 40. Parallels, also weit südlich der Zentralalpen. Bis nach Hokkaido ist neuerdings durch Minato (1955) Mammonteus primigenius nachgewiesen.

Die Südgrenze der borealen Nadel- gegen die anspruchsvolleren Mischwälder wird man demzufolge entsprechend den Darlegungen von Wissmanns (1938) in der Südmandschurei mindestens bei 42 bis $43^{\circ}$ Nord annehmen müssen.

Faßt man das Ergebnis der Ubersicht über den Verlauf der würmzeitlichen klimabedingten Vegetationszonen in den verschiedenen Teilen Eurasiens etwas schematisiert zusammen und vergleicht es mit der gegenwärtigen Lage der Südgrenzen von 'Tundra und borealem Nadelwald als wichtigsten physiogeographischen Landschaftsgliedern, so ergibt sich das Bild, daß zwischen der exzessiven Südverlagerung der physiogeographischen Zonen auf der atlantischen Westseite des Kontinentes und einer etwas geringeren, aber doch sehr deutlichen auf seiner pazifischen Ostseite im kontinentalen zentralen Teil eine minimale Verlagerung während der letzten Kaltzeit stattgefunden hat. Es ist jener Raum von Südwest- und Mittelsibirien, für den Guerassimow \& Markow die eingangs zitierte Feststellung getroffen haben.

Welche Folgerungen lassen sich nun aus diesem Tatbestand hinsichtlich der kaltzeitlichen Temperaturverhältnisse im zentralen Eurasien ziehen?

Im globalen Überblick wird die durchschnittliche kaltzeitliche Depression des Breitenkreismittels der Jahrestemperatur der bodennahmen Luftschichten der Breiten $50^{\circ}$ von verschiedenen Autoren, zuletzt FloHN (1953), übereinstimmend auf 7 bis $8^{\circ} \mathrm{C}$ geschätzt. Dabei ist die Erniedrigung der Wintertemperatur größer, die der Sommertemperatur kleiner als die des Jahresmittels (vielleicht 4 bis $5^{\circ}$ im Hochsommer, 11 bis $12^{\circ}$ im Hochwinter). In die Mittelbildung gehen einerseits die ozeanischen Räume, andererseits die kontinentalen ein. Nun müssen auf Grund der fundamentalen allgemeinklimatologischen Erfahrung, nach der in der Breiten polaufwärts von $40^{\circ} \mathrm{Br}$., in denen „die Wintertemperatur den Charakter der Jahrestemperatur bedingt“ (HANN-SüRING 1939, S. 178), die kontinentalen Gebiete diejenigen minimaler Wintertemperatur und im Vergleich zu den ozeanischen Teilen auch relativ niedriger Jahrestemperatur sind, in den ozeanischen Gebieten notwendig die Drepressionen der Jahresmittel- und Wintertemperatur geringer, in den kontinentalen merklich größer als das Breitenkreismittel gewesen sein, wenn nicht eine gegenüber der Gegenwart stark abgeänderte Zirkulationsanordnung diese Regel, die für die rezent vorherrschende Zonalzirkulation (High-zonal-index-Typ WILIEtrT's 1950) gilt, dynamisch modifiziert hat. FLOHN gibt die Depression der Jahresmitteltemperatur für die ozeanischen Gebiete mit 3 bis $5^{\circ} \mathrm{C}$ an. Die Sommertemperatur muß in den eisnahen Gebieten Mitteldeutschlands nach verschiedenen Ableitungen (vgl. WEISCHET 1954) um 10 bis $12^{\circ}$, die Wintertemperatur um $16^{\circ}$ zurückgegangen sein. Und für das Gebiet um Laibach hat Poser (1947) als für die würmzeitliche Baumgrenze Werte von etwa $10^{\circ}$ bzw. $12^{\circ}$ überzeugend abgeleitet.

Um eine entsprechende Vorstellung von der unter den vorausreferierten physiogeographischen Bedingungen zur Würmeiszeit möglichen Temperaturdepression in Südwest- und Mittelsibirien zu bekommen, habe ich die gegenwärtigen thermischen Verhältnisse mitein- 


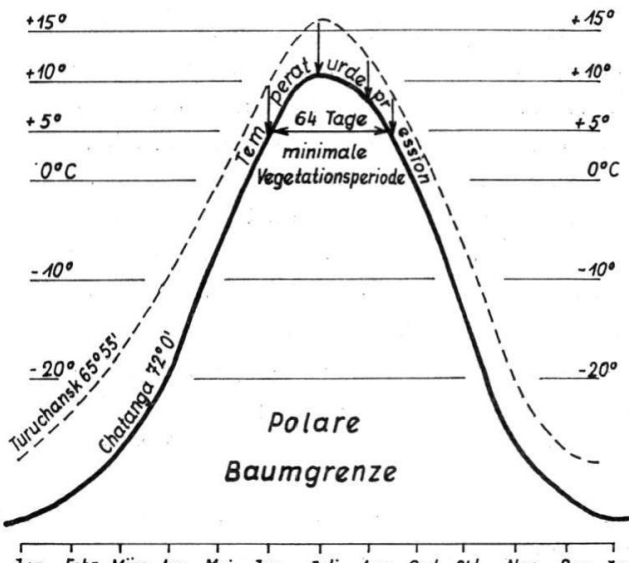

Jan. Febr. März Apr. Mai Jun. Juli Aug. Sept. Okt. Nov. Dez. Jan.

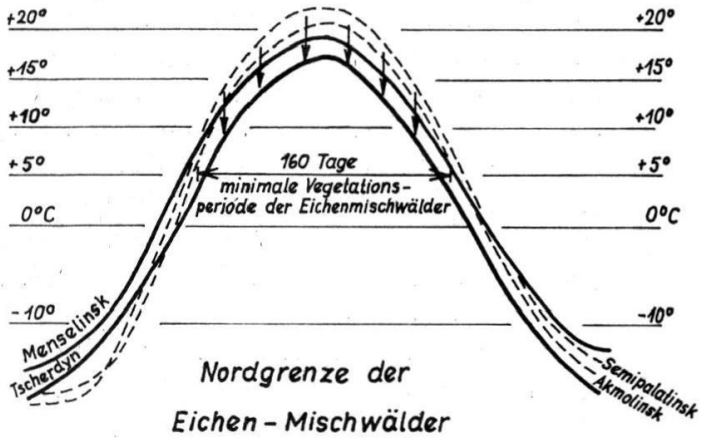

Jan. Febr. März Apr. Mai Jun. Juli Aug. Sept. Okt. Nov. Dez.

Abb. 2. Vergleich des Temperaturganges im Bereich der rezenten und würmeiszeitlichen a) polaren Baumgrenze

b) Nordgrenze der Laubmischwälder und die mögliche sommerliche Temperaturdepression während der Würmeiszeit in Westsibirien. ander verglichen, die jeweils in den Bereichen der rezenten und der kaltzeitlichen polaren Verbreitungsgrenze der Nadelbäume der Taiga einerseits und der anspruchsvolleren Laubbäume der Mischwaldzone andererseits herrschen (vgl. Abb. 2). Dabei wurden als Begrenzungswerte nicht allein die Monatsmitteltemperaturen, sondern auch die verschiedenen Längen der Vegetationsperioden berücksichtigt. (Letztere läßt sich ungefähr durch die mittlere Zeitspanne festlegen, welche Tagesmitteltemperaturen über $5^{\circ} \mathrm{C}$ aufweist; für das Verfahren spielt es kaum eine Rolle, einen anderen Wert zwischen 5 und $0^{\circ}$ zu nehmen). Monatsmittel der Lufttemperatur für die Sommermonate und Zahl der Tage über der Grenztemperatur der Vegetationsperiode sind als die zwei Größen anzusehen, welche die Anordnung der subpolaren Vegetationszonen von der thermischen Seite wesentlich bestimmen. Die Zahl der Tage über $5^{\circ} \mathrm{C}$ läßt sich aus dem Sowjetatlas (1937) entnehmen, die Monatsmittel aus den von Köppen (1939) zusammengestellten Klimawerten für Rußland.

Zunächst die polare Baumgrenze (Abb. 2 a). Bei der relativ geringen Stationsdichte ist es zwar schwer, jeweils Stationen aus dem engsten Bereich der derzeitigen und würmzeitlichen polaren Baumgrenze zu bekommen. Doch Chatanga $(50 \mathrm{~m}+\mathrm{NN})$ in Mittel- und Kasetschje $(17 \mathrm{~m}+\mathrm{NN})$ in Ostsibirien repräsentieren ziemlich genau die Tundrengrenze. Rund 64 bis 70 Tage über $5^{\circ} \mathrm{C}$ bei einem Julimittel von 10 bis $11^{\circ}$ sind die Kennzeichen. Setzt man diese Bedingungen auch für die würmzeitliche polare Baumgrenze an und vergleicht damit die im Gebiet ihrer damalagen Lage gegenwärtig herrschenden thermischen Verhältnisse, so kann nahe der Mündung der Unteren Tunguska die würmzeitliche Temperaturdepression während der Sommermonate nur die Größenordnung von 4 bis $6^{\circ} \mathrm{C}$ gehabt haben. Dort ist nämlich in Turuchansk $\left(65^{\circ} 55^{\prime}, 40 \mathrm{~m}+\mathrm{NN}\right)$ heute das Julimittel ungefähr $16^{\circ}$ bei einer Dauer der V'egetationsperiode von rund 100 Tagen. In Mittelsibirien ist die zahlenmäßige Abschätzung aus Ermangelung von Beobachtungsstationen im Zentralsibirischen Plateau nicht möglich. Aber die Werte des Temperaturrückganges müssen geringer sein als weiter westlich, da rezente und eiszeitliche Tundrengrenze fast zusammenfallen.

Für die Errechnung der Temperaturdepression im Gebiet der eiszeitlichen Nordgrenze der Laubmischwälder (s. Abb. 2 b) ergibt sich die Schwierigkeit, daß gegenwärtig Laubwälder, an denen die Eiche beteiligt ist, wegen der nach Osten zunehmenden Trockenheit auf das Gebiet westlich vom Ural beschränkt sind. Die Verbreitungskarten im Sow jetatlas (1937) unterscheiden „Nadel- und breitblättrige Wälder (Mischwälder) sowie 
„breitblättrige Wälder (Laubwälder mit Eiche)“. Nach Westsibirien reichen nur „Espenund Birkenwälder". Als minimale thermische Voraussetzung der Laubwälder muß ein Temperaturgang angesehen werden, welcher $\mathrm{z}$ wischen denjenigen der Stationen Tscherdyn (gelegen bei $60^{\circ} 24^{\prime}, 177 \mathrm{~m}+\mathrm{NN}$ am Südsaum innerhalb der Nadelwaldzone) und Menselinsk (55 $39^{\prime}, 80 \mathrm{~m}+\mathrm{NN}$ im nördlichen Bereich der Laubwälder) verläuft (ausgezogene Linien in Abb. 2 b). Dieser Temperaturgang entspricht für das Sommerhalbjahr demjenigen in der breitenmäßig vergleichbaren Zone des Westsibirischen Tieflandes am Unteren Irtysch (Station Tara z. B.), in der heute aus hygrischen Gründen die Eiche fehlt. Die zugehörige Vegetationsperiode muß in Westsibirien mit wenigstens 160 Tagen angesetzt werden, da die Dauer an der Nordgrenze der Laubwälder von 185 Tagen bei Kiew mit wachsender Kontinentalität auf 170 Tage an der Wolga und 165 Tage südlich Perm abnimmt. Weitere 5 Tage wurden für das noch sommerwärmere Westsibirien in Rechnung gestellt.

Den gegenwärtigen Temperaturgang für diejenigen Gebiete, in denen nach FRENZEL \& TROLL (1952) in der letzten Kaltzeit Laub-Mischwälder mit Eichen vorhanden waren, repräsentieren die Werte von Akmolinsk $\left(51^{\circ} 10^{\prime}, 347 \mathrm{~m}+\mathrm{NN}\right)$ und Semipalatinsk $\left(50^{\circ} 24^{\prime}, 207 \mathrm{~m}+\mathrm{NN}\right)$, wobei die zuletzt genannte Station sicher thermisch günstiger ist als die Standorte würmzeitlicher Laub-Mischwälder (gestrichelte Kurven in Abb. 2 b). Aus dem Vergleich ergibt sich, daß die sommerlichen Temperaturdepression im Bereich zwischen oberem $\mathrm{Ob}$ und Irtysch in der Baraba-Steppe höchstens 3 bis $5^{\circ} \mathrm{C}$ betragen haben kann. Nach der Wahl der Stationen sind dieses maximale Abschätzungswerte, die wahrscheinlichsten liegen zwischen 2 und $4^{\circ} \mathrm{C}$.

Als Resultat für den Gesamtraum des zentralen Eurasiens ist festzustellen, daß auf Grund der gegenwärtigen Vorstellung vom Verlauf der kaltzeitlichen Vegetationszonen die Sommertemperatur zur letzten Eiszeit nahe dem Polarkreis maximal 5 bis $8^{\circ}$, in Breiten um $50^{\circ} 2$ bis $4^{\circ}$ niedriger gewesen sein kann als in der Gegenwart. Im Vergleich zum ozeanischen Westen ist also die Temperaturdepression im Innern des Kontinentes während des Sommers in den höheren Breiten 4 bis $5^{\circ}$ (verglichen mit Mitteldeutschland), bei ca. $50^{\circ} \mathrm{N} 6$ bis $8^{\circ}$ (verglichen mit dem Raum Laibach) geringer gewesen. Die Differenz zum Breitenkreismittel in $50^{\circ}$ beträgt 1 bis $2^{\circ}$.

Wenn man das als Folge der verschieden großen Kontinentalität ansieht, so verlangt die Anwendung eben dieses Klimafaktors aber nach der vorher zitierten klimatologischen Grunderfahrung auch, daß man die Depression von Winter- und Jahresmitteltemperatur als größer annimmt als im ozeanischen Westen oder im Breitenkreismittel.

Die Wintertemperatur war in den periglazialen Bereichen Mitteldeutschlands um 16 im Raume um Laibach ca. $12^{\circ}$, im Breitenkreismittel um 10 bis $11^{\circ}$ zurückgegangen. Veranschlagt man nun den Kontinentalitätseffekt für den Winter mit derselben Größenordnung temperatur-erniedrigend, mit der er Sommer erhöhend wirksam wurde, also mit 4 bis 5 bzw. 6 bis $8^{\circ}$, so wird man die Depression der Wintertemperatur im Gebiet um Turuchansk mit ( -16 zuzüglich 4 bis $5^{\circ}$ ) 20 bis $21^{\circ}$, im Bereich der Baraba-Steppe mit $\left(-12\right.$ zuzüglich 6 bis $\left.8^{\circ}\right) 18$ bis $20^{\circ}$ annehmen dürfen, abgeleitet von der Erniedrigung der Wintertemperaturen im ozeanischeren Mitteleuropa. Ausgehend von der Erniedrigung des Breitenkreismittels der Wintertemperatur für $50^{\circ}$ Nord (11 bis $12^{\circ}$ nach FLOHN 1953), ergibt sich für das Gebiet der würmzeitlichen Laub-Mischwaldgrenze ein Rückgang der Wintertemperatur um (11 bis $12^{\circ}$ zuzügl. Kontinentalitätseffekt von 1 bis $\left.2^{\circ}\right) 12$ bis $14^{\circ}$. $\mathrm{Da}$ Turuchansk heute schon ein minimales Monatsmittel von minus $28^{\circ}$ hat, müßten zur Kaltzeit 48 bis $49^{\circ}$ erreicht worden sein. Im Gebiet der Baraba-Steppe, wo heute im Januarmittel 16 bis $17^{\circ}$ unter Null gemessen werden, wären 35 bis $36^{\circ}$, bzw. wenigstens $29^{\circ}$ (nach dem Breitenkreismittel errechnet) unter dem Gefrierpunkt anzunehmen.

Bei den vorher abgeleiteten Julitemperaturren von $12^{\circ}$ an der polaren Baumgrenze und 16 bis $17^{\circ}$ an der Laub-Mischwaldgrenze, ergeben sich somit Jahresamplituden von 
ungefähr $60^{\circ} \mathrm{C}$ um Turuchansk, sowie zwischen 45 und $56^{\circ} \mathrm{C}$ im Gebiet zwischen Akmolinsk und Semipalatinsk. Es resultieren also folgende thermischen Kennzeichen:

\begin{tabular}{ccc}
\multicolumn{2}{c}{ Monatsmitteltemperatur } & Jahresamplitude \\
des Hochsommer- & des Hochwinter- & \\
$+12^{\circ}$ monats & $-48 / 49^{\circ}$ & ca. $60^{\circ} \mathrm{C}$ \\
+16 bis $17^{\circ}$ & -29 bzw. $35 / 36^{\circ}$ & $45 / 46$ bzw. $52^{\circ} \mathrm{C}$
\end{tabular}

polare Waldgrenze

(untere Tunguska)

Nordgrenze des Laubmischwaldes $\quad+16$ bis $17^{\circ} \quad-29$ bzw. $35 / 36^{\circ} \quad 45 / 46$ bzw. $52^{\circ} \mathrm{C}$

(Baraba-Steppe)

Diese Werte ergeben sich notwendig aus der prämissenhaften Anwendung der These, daß die „klimatische Stabilität" eine Folge der kontinentale Lage ist. Es läßt sich so aus dem Ergebnis der Ableitung die Berechtigung der Prämisse prüfen. Grundsätzlich muß man die Möglichkeit einräumen, daß im östlichen Westsibirien die kalkulierten Temperaturamplituden aufgetreten sein können, da Jahresamplituden von $65^{\circ} \mathrm{bzw} .55^{\circ}$ heute in entsprechenden Breiten vorkommen. Aber sie finden sich gegenwärtig erst 30 bis 40 Längengrade weiter ostwärts; nahe dem Polarkreis erst im Lenabogen, in $50^{\circ}$ Breite am oberen Tschilka, d.h. in unmittelbarer Nähe der Räume größter thermischer Kontinentalität überhaupt. Es ist also festzustellen, daß nicht das gegenwärtige Ausmaß der Kontinentalität in West- und Mittelsibirien diesem Raum zur Stabilität der physiogeographischen Bedingungen verholfen hat, daß dazu vielmehr in ihm eine Verstärkung der kontinentalen Faktoren notwendig war.

Diese Intensivierung der Kontinentalitätsfaktoren in Westsibirien muß mit großer Wahrscheinlichkeit als Ergebnis einer Westverlagerung der Räume größter Kontinentalität im Vergleich zur aktuellen Anordnung angesehen werden, wie der Umstand zeigt, daß die physiogeographische Veränderung im Ostteil Eurasiens wieder größer waren als im mittleren Teil. Bei Voraussetzung der regionalen Klimadifferenzierung der Gegenwart läßt sich nämlich schwerlich erklären, daß ostwärts des Werchojanischen Gebirgsbogens cine beträchtlich stärkere Eisakkumulation stattgefunden hat, da in der Gegenwart in erheblicher Ausdehnung in Ostsibirien sowohl die Niederschläge als auch die Schneedeckenhöhen noch geringer sind als weiter westlich. Erst im unmittelbaren Einflußbereich des Bering-Meeres wachsen die genannten Elementewerte wieder an (vgl. Sowjetatlas S. 112-114). Und die Tatsache, daß die Südverschiebung der Grenzen von Tundra, Nadel- und Labwaldzone im Gebiet größter Kontinentalität größer war als weiter westlich, steht im Widerspruch zu dem vorher prämissenhaft angenommenen Prinzip, daß thermische Kontinentalität stabilisierend wirkt.

Diese Hinweise führen u. E. notwendig zur Annahme einer räumlich verschiedenen Anordnung des Kontinentalitätsfaktors zur Eiszeit im Vergleich zu gegenwärtigen Bedingungen, zu der Annahme einer Westverlagerung der Gebietes größter Kontinentalität. Diese kann grundsätzlich zwei Ursachenkomplexe haben: entweder eine Anderung der Land/Wasser-Verteilung oder eine solche des vorherrschenden Zirkulationsregimes. Der erstere war zweifellos wegen der Absenkung des Meeresspiegels und dem Aufbau der kontinentalen Eisschilde im westlichen Eurasien gegeben. Daß er aber über den ganzen Doppelkontinent allein für sich die vorauf dargelegte tiefgreifende Wirkung ausübte, ist unwahrscheinlich. Erstens ist der Zuwachs der Landmasse im Westen Europas im Vergleich zur Westverlagerung des Kontinentalitätsmaximums gering. Und zweitens ist so bei Erhaltung vorwiegender Zonalzirkulation nicht die Verlagerung des Gebietes größter Kontinentalität von Ost- nach Zentralsibirien zu verstehen. Dafür kann nur eine Änderung der gegenwärtig über Eurasien herrschenden allgemeinen atmosphären Zirkulation eine befriedigende Erklärung liefern.

So führt m. E. die konsequente Anwendung der Prämisse, daß die Stabilität der Klimabedingungen in Mittel- und Westsibirien eine Folge der Kontinentalität dieses Raumes sei, zu der Folgerung, daß eine Änderung des gegenwärtigen meteorologischen Regimes wenigstens zusätzlich angenommen werden muß. 


\section{Einfluß der Zirkulationsänderung auf die Konstanz der thermischen Bedingungen}

Vorstellungen über die Zirkulationsänderung sind unabhängig von den vorauf diskutierten Ergebnissen auf Grund der räumlichen Lage der großen Eisakkumulationen entwickelt worden, und es erhebt sich die Frage, wie diese sich zu den angeführten Folgerungen für West- und Mittelsibirien verhalten.

FLOHN (1952) hat an Hand zirkumpolarer Höhenluftdruckkarten die aktuell anormale Zirkulation, die zu außergewöhnlich schneereichen und kalten Winterperioden in den Gebieten der ehemaligen pleistozänen Eisansammlungen führten, mit derjenigen des normalen Winterablaufes verglichen. Er kommt zu dem Ergebnis, daß der Meteorologe in der Verteilung der pleistozänen Vergletscherungszentren „ein fossiles Abbild der Höhenströmung und damit der mittleren troposphärischen Temperaturverteilung in der Eiszeit sehen kann". Die Aussage beruht auf der Tatsache, daß die aktuelle synoptische Situation schneedeckenreicher Ausnahmezeiten in charakteristischer Abwandlung der normalen Höhendruckverteilung dadurch gekennzeichnet ist, daß sich über den Gebieten der pleistozänen Vereisungszentren kräftige Tieftrucktröge des Zirkumpolarwirbels finden. Die eiszeitliche Höhenströmung unterschied sich demnach von der gegenwärtigen dadurch, daß erstens die Höhentröge des Tiefdruckwirbels kräftiger ausgebuchtet und zweitens etwas nach Westen verschoben waren. (Vgl. auch Abb. 128 und die entsprechende Darstellung bei Woldstedt 1954). Der das rezente Klima beherrschende Trog über Nordamerika war von der Ostküste über das Gebiet der Hudson-Bay und der Großen Seen gewandert, der gegenwärtig im Mittel über Westsibirien nur schwach angedeutete in kräftiger Form nach Europa gerückt. Zwischen den beiden lag über dem Atlantik der entsprechende Höhenhochdruckkeil. Dadurch wurde die das rezente Klima des Westteiles Eurasiens beherrschende durchgehende Warmluftdrift der Westwindzone über dem Atlantik blockiert, die Luftmassen im statistischen Mittel auf der Westseite des Hochdruckkeils bis in subpolare Breiten geführt, von wo die Ausgleichsströmung Europa als nördliche Kaltluftdrift auf der Ostseite des Hochdruckkeils erreichte. Als zentrale Bedingung für die Ausbildung einer Vereisung Westeurasiens wird also angesehen, daß dieser Teil des Kontinentes aus der Vorderseiten-Warmluftzufuhr in den Rückseiten-Kaltluftstrom gelangt, wie es z.B. die Abb. 7 bei Flohn angibt. Diese Situation ist Teil einer erhöhten Meridionalzirkulation, des „low zonal index-Typ“, wie WILlett (1950) ihn nennt.

Als Stütze der Ableitung FlohNs an Hand der Großwetterlage im Dezember 1950 kann übrigens gelten, daß 29 unabhängig ausgewählte Testfälle über die synoptischen Bedingungen einer Schneedeckenbildung im normalerweise schneefreien Westdeutschen Flachland ganz eindeutig zu dem Ergebnis führten, daß die episodisch ausnahmsweise Ansammlung von Schnee in dem genannten Bereich eine Blockierung der Westdrift über dem Atlantik und Ersatz durch eine meridionale Nordsteuerung zwischen einem Hochdruckkeil über dem östlichen Atlantik und einem Tiefdrucktrog über dem mittleren Europa erforderte (WeIsCHet 1948).

Uber dem eurasiatischen Doppelkontingent wiederholt sich dann dic mäanderförmige Druckanordnung, wie sie für den Bereich des Atlantiks und seiner angrenzenden Festlandgebiete referiert wurde. Die relativ große Vergletscherung des Ostsibirischen Raumes ergab sich im Bereich des dort auftretenden nächsten Höhentroges. Zwischen diesem und demjenigen über dem mittleren Europa muß sich aus dynamisch-meteorologischen Gründen ein Hochdruckkeil einschalten, der gerade den Raum größter Stabilität der Klimabedingungen im zentralen Teil Eurasiens beeinflußt. FLOHN (1952) hat seine Folgen schon angedeutet, indem er als Nebenkonsequenz der eiszeitlich verstärkten Meridionalzirkulation die Notwendigkeit gesteigerter Warmluftzufuhr über dem Atlantik, dem Pazifik 
und in schwächerem Maße auch über dem hier in Frage stehenden Mittelsibirien herausstellte.

Um diese Notwendigkeit für den in Frage stehenden Raum speziell zu überprüfen und zu untermauern, habe ich mir für die Wintermonate der Jahre 1949 bis 1958 aus den Täglichen Wetterkarten des Deutschen Wetterdienstes und den zirkumpolaren Synoptic Weather Maps des US-Weather Bureaus eine Ubersicht über die in den betreffenden Monaten vorherrschenden Großwetterlagen und die mit ihnen im atlantischen Westen bzw. in Westsibirien verbundenen Temperaturanomalien verschafft. Aus dieser ergibt sich, daß bei längerer Erhaltung eines Höhentroges über der Mitte Europas regelhaft einerseits im Bereich des Troges zu kalte, schneereiche Winterperioden auftreten, während sich gleichzeitig in Westsibirien als Folge der Warmluftzufuhr aus Südwesten positive Temperaturanomalien einstellen. Als Beispiele können außer der von FLOHN (1953) herangezogenen Dezemberlage 1950 kürzere Zeitabschnitte der Monate Januar, Februar und November 1952, des Januars und Februars 1955 sowie des Februars 1956 dienen. Außerdem sind in der nachstehenden Tabelle diejenigen Hochwintermonate zwischen 1941 und 1952 aufgeführt, die an den Stationen Barnaul bzw. Swerdlowsk besonders große positive Temperaturanomalien aufwiesen (die freundliche Vermittlung der in Veröffentlichungen nicht zugänglichen Werte verdanke ich Frau Prof. E. RuBinsteIN, Leningrad).

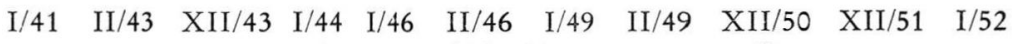
Mittlere monatliche Temperaturanomalie

\begin{tabular}{|c|c|c|c|c|c|c|}
\hline Barnaul & $+4,0^{\circ}$ & $+3,7^{\circ}$ & $+3,4^{\circ}$ & $\begin{array}{l}+7,7^{\circ}+6,2^{\circ} \\
+10^{\circ}(16 .-28 .)\end{array}$ & & $\begin{array}{l}+8,7^{\circ}+3,3^{\circ} \\
\quad+5^{\circ}(16 .-28 .)\end{array}$ \\
\hline Swerdlowsk & $+2,3^{\circ}$ & $+4,3^{\circ}$ & $+5,9^{\circ}+4,6^{\circ}$ & $\begin{array}{r}+4,0^{\circ}+8,8^{\circ} \\
+7^{\circ}(16 .-28 .) \\
\text { Zirkulationstyp }\end{array}$ & $+5,6^{\circ}$ & $+6,6^{\circ}$ \\
\hline
\end{tabular}

\begin{tabular}{|c|c|c|c|c|c|c|c|c|c|c|}
\hline \multirow{4}{*}{ Frankfurt/M. } & \multicolumn{10}{|c|}{ Zahl der Schneedeckentage } \\
\hline & 27 & 0 & 1 & 1 & 1 & 6 & 3 & 0 & 24 & 6 \\
\hline & \multicolumn{10}{|c|}{ Temperaturanomalie } \\
\hline & $-3,6$ & $+2,3$ & $-0,4$ & $+3,4$ & $-1,9$ & $\begin{array}{l}-1, \\
(20 .\end{array}$ & -1,7 & $+0,3$ & $-2,4$ & $+1,0-0,3(16 .-30)$. \\
\hline
\end{tabular}

Auf Grund der o. a. synoptischen Übersicht stellen sich die Anomalien im Januar und Februar 1949 sowie im Dezember 1951 als Folge einer außergewöhnlich starken Zonalzirkulation heraus, bei der auch im atlantischen Westen zu hohe Wintertemperaturen und geringe Schneefälle auftraten, wie die angeführten Werte für Frankfurt/M. (Mollwo 1958) belegen. Sie scheiden aus der Betrachtung aus, da nur die Fälle zu kalter Winter im atlantischen Bereich interessieren. Der Januar 1952 ist dadurch ausgezeichnet, daß sich in der 2. Monatshälfte eine Trogsteuerung über Mitteleuropa einstellte, die in Frankfurt ab 18. zu negativen Temperaturanomalien bis zu 1,3 Grad im 6. Pentatenmittel und zu sechs Schneedeckentagen führte, während in Swerdlowsk auf der Ostseite des Troges zwischen den 16. und 28. eine im Durchschnitt 5,2 Grad zu hohe Temperatur herrschte. Die Großwetterlage des Dezembers 1950 ist durch die Höhendruckverteilung in Abb. 7 bei Flohn (1953) schon als Typ der Meridionalzirkulation bekannt. Die positive Temperaturanomalie betrug in Swerdlowsk 5,6 $6^{\circ}$ während Frankfurt im gleichen Monat eine Negative von $2,4^{\circ}$ bei der extrem langen Schneedeckendauer von 24 Tagen hatte. Ein ähnliches Beispiel dürfte der Januar 1941 sein, wenn dafür auch das synoptische Material noch nicht zur Verfügung stand. Die außerdem angeführten Werte für kürzere Zeitabschnitte im Januar und Februar 1946 sowie im Dezember 1943 fügen sich in das Bild gut ein. Man findet so also durchaus die Regel bestätigt, daß die vorherrschende Meridionalzirkulation im atlantischen Westen Eurasiens zu kalten und schneereichen Wintern 
führt, im mittleren Teil des Kontinentes zwischen Ural und mittelsibirischem Bergland dagegen den umgekehrten Effekt, nämlich relativ milde Winter zur Folge hat. Nimmt man nun wie FloHN (1953) die Meridionalzirkulation als eine meteorologische Voraussetzung zur Ausbildung der Vereisung, so ist mit ihr notwendig verbunden, daß in Westund Mittelsibirien die Klimaverschlechterung im Vergleich zu den im Westen und Osten angrenzenden Räumen des Doppelkontinentes relativ gering gewesen sein muß. Der Vorderseiten-Warmlufteinfluß milderte die weltweite primäre und sekundäre eiszeitliche Temperaturerniedrigung, während im atlantischen Westen und in Ostsibirien die nördliche Luftzufuhr sich verstärkte.

Dadurcherscheintdie Stabilitätder Klimabedingungen Westund Mittelsibiriens in anderem Licht. Sie muß m. E. als zirkulationsbedingt derart angesehen werden, daß der Luftmassentransport in diesem Raum im Interglazial bei verstärkter Zonalzirkulation subpolare Akzente, bei derim Glazial vorherrschenden Meridionalzirkulation subtropische Akzente bekommt. Diese wirkèn der primären, zirkulationsunabhängigen Temperaturwelle zwischen Interglazial und Glazial entgegen und schwächen ihre Amplitude, d. h. führenzu relativ konstanten thermischen Bedingungen.

\section{Literatur:}

Blagowetschtschenskaja, M. N.: Spuren der Vereisung im Tal der Unteren Tunguska (Ostsibirien) und im Gebiet ihrer Nebenflüsse (russ.). - Materialy Wsessojasn. nautsch. issled. geol. Inst. 7, S. 243-248, 1955. (Ref. Mirtsching, Zentralbl. f. Geol. u. Min. 1958/I).

BüDEL, J.: Die Klimazonen des Eiszeitalters. - Eiszeitalter u. Gegenw. 1, S. 16-26, 1951.

Deutscher Wetterdienst: Täglicher Wetterbericht.

FloHN, H.: Allgemeine atmosphärische Zirkulation und Paläoklimatologie. - Geol. Rd. 40, S. 153 bis 178, Stuttgart 1952. - - Studien über die atmosphärische Zirkulation in der letzten Eiszeit. - Erdkunde 17, S. 266-275, Bonn 1953.

Frenzel, B., \& Troll, C.: Die Vegetationszonen des nördlichen Eurasiens während der letzten Eiszeit. - Eiszeitalter u. Gegenw. 1, S. 154-167, 1952.

Guerassimow, I. P., \& Markow, V. V.: La paléogéographie des territoires de L’U.R.S.S. durant la période glaciaire. - C. R. Congr. Géol. Int. Alger 1952, fasc. XV, S. 289-304, Alger 1954.

Hann-Süring: Lehrbuch der Meteorologie, Bd. I. Leipzig 1939.

KöpPEN, W.: Klimakunde von Rußland in Europa und Asien. - Hdb. d. Klim. Bd. III, Teil N, Berlin 1939.

Mrnato, M.: Zu den Mammonteusfaunen Hokkaidos. - Jap. J. of Geol. a. Geogr. 26, S. 105-113, 1955.

Mollwo, H.: Klimawerte von Frankfurt/Main 1857-1956. - Ber. Dt. Wetterd. Nr. 43, 1958.

Nikolskaja, W. W., \& Schtscherbakow, I. N.: Spuren einer alten Vereisung im Gebirge Tüküringa-Dshangdy (russ.) - Iswestja Ak. Nauk. SSSR., Ser. geogr., Nr. 2, S. 58-65, 1956. (Ref. MirTsching, Zentralbl. Geol. u. Min. 1957/I.)

Parmuzin, J. P.: Zur Paläogeographie Mittelsibiriens während des Quartärs (russ.). - Voprosy Geogr. 35, S. 82-111, 1954. (Ref. Mirtsching, Zentralbl. f. Geol. u. Min. 1956.)

Poser, H.: Dauerfrostboden und Temperaturverhältnisse während der Würm-Eiszeit. - Naturwiss. 34, S. 10-18, 1947. - - Boden- und Klimaverhältnisse in Mittel- und Westeuropa während der Würmeiszeit. - Erdk. 2, S. 53-68, Bonn 1948. - - Auftautiefe und Frostzerrung im Boden Mitteleuropas während der Würmeiszeit. - Naturwiss. 34, S. 232-238, 262-267, Berlin 1947.

Sowjetatlas: Moskau 1937.

ThIEL, E.: Die Eiszeit in Sibirien. - Erdk. 5, S. 16-35, Bonn 1951.

US Weather Bureau: Synoptic Weather Map, Daily Series, Part. I, 1950-52.

Weischet, W.: Die Schneedeckenverhältnisse im Rheinischen Schiefergebirge und ihre synoptischmeteorologischen Bedingungen. - Diss. Bonn 1948 (Zusammenfassung „Decheniana“, Verh. d. nat. hist. Ver. Rheinl. u. Westf. 1949.) - - Die gegenwärtige Kentnis vom Klima in Mitteleuropa beim Maximum der letzten Vereisung. - Mitt. geogr. Ges. München 39, S. 95-116, 1954. 
WiLlett, H. C.: The general circulation at the last (Würm) glacial maximum. - Geogr. Ann. 31, Stockholm 1950.

von WIssmanN, H.: Über Lößbildung und Würmeiszeit in China. - Geogr. Z. 44, S. 201-220, Leipzig 1938.

Woldstedt, P.: Das Eiszeitalter, Bd. I. Stuttgart 1954.

Zeuner, F. E.: Das Problem der Pluvialzeiten. - Geol. Rd. 41, S. 242 ff., Stuttgart 1953.

Manuskr. eingeg. 11. 6. 1960.

Anschrift des Verf.: Dr. W. Weischet, z. Zt. Universidad Austral de Chile, Instituto de Geografia, Valdivia/Chile, Casilla 1020. 